\title{
Downregulation of T-Cell Transcription Factors in Adult Latent Autoimmune Diabetes with High-Titer Glutamic Acid Decaroxylase Antibody
}

\author{
Xia Wang · Lin Yang • Ying Cheng · Huiying Liang · Jingping $\mathrm{Hu}$ • \\ Peilin Zheng · Gan Huang · Zhiguang Zhou
}

Received: January 21, 2019 / Published online: March 20, 2019

(C) The Author(s) 2019

\begin{abstract}
Introduction: Latent autoimmune diabetes in adults (LADA) shows a heterogeneous clinical profile that is dependent on the glutamic acid decaroxylase antibody (GADA) titer. We speculated that LADA patients with a high or low GADA titer may have distinct T-lymphocyte subset profiles and distinct expression patterns of transcription factors involved in T-cell immunomodulation.

Methods: Patients with LADA $(n=40)$ and type 2 diabetes (T2DM; $n=14$ ) were recruited to the study, and peripheral blood mononuclear cells were isolated. The proportions of T-lymphocyte subsets (Th1 [T helper type 1], Th2 [T helper
\end{abstract}

Xia Wang and Lin Yang contributed equally to this work.

Enhanced Digital Features To view enhanced digital features for this article go to https://doi.org/10.6084/ m9.figshare.7798157.

X. Wang - L. Yang - Y. Cheng - H. Liang - J. Hu . P. Zheng · G. Huang · Z. Zhou (ه)

Department of Metabolism and Endocrinology, The Second Xiangya Hospital, Central South University, Changsha, Hunan, China

e-mail: zhouzhiguang@csu.edu.cn

X. Wang - L. Yang - Y. Cheng - H. Liang · J. Hu . P. Zheng · G. Huang · Z. Zhou

Key Laboratory of Diabetes Immunology (Central South University), Ministry of Education, National Clinical Research Center for Metabolic Diseases, Changsha, Hunan, China type 2], Treg [regulatory T], and Th17 [T helper type 17] cells) were determined by flow cytometry. Real-time polymerase chain reaction (PCR) was performed to estimate mRNA expression levels of the T-cell subtype-enriched transcription factors T-bet (Th1), GATA3 (Th2), transcription factor forkhead box protein 3 (FOXP3) (Treg), and RORC (Th17).

Results: The frequency of Th1 (as a percentage of total $\mathrm{CD} 4^{+} \mathrm{T}$ cells) was greater in the LADA patients with high-titer GADA than in the LADA patients with low-titer GADA $(11.06 \pm 1.62$ vs. $7.05 \pm 0.86, P=0.030)$. Compared to the T2DM group, in the low-titer GADA group the frequency of Th1 was significantly reduced (7.05 \pm 0.86 vs. $16.75 \pm 3.73, P=0.017)$ and the frequency of Th17 frequency was signficantly increased $(1.11 \pm 0.09$ vs. $0.74 \pm 0.16$, $P=0.017)$. Compared to T2DM patients, in the high-titer GADA group there was a significantly reduced expression of FOXP3 (0.35 \pm 0.13 vs. $1.75 \pm 0.54, P=0.002)$, RORC $(0.53 \pm 0.19$ vs. $2.00 \pm 0.77, P=0.046)$, and GATA3 $(0.74 \pm 0.17$ vs. $2.31 \pm 0.91, P=0.046)$. Similarly, the hightiter GADA group expressed reduced levels of FOXP3 and RORC compared to the low-titer GADA group $(0.35 \pm 0.13$ vs. $1.50 \pm 0.41$, $P=0.027 ; \quad 0.53 \pm 0.19 \quad$ vs. $\quad 1.35 \pm 0.21$, $P=0.027$, respectively). There was a negative correlation between FOXP3 expression level and GADA titer for the entire cohort $(r=-0.0433$, $P=0.015)$ and a stronger negative correlation in LADA patients $(r=-0.606, P=0.008)$. 
Conclusion: LADA patients with high-titer GADA express lower levels of T-cell transcription factors, including the Treg transcription factor FOXP3, which may contribute to differences in the clinical profile compared to LADA patients with low-titer GADA.

Trial Registration: ClinicalTrials.gov identifier, NCT01159847.

Keywords: Glutamic acid decarboxylase antibody; Latent autoimmune diabetes in adults; $\mathrm{T}$ lymphocyte subsets; Transcription factors

\section{INTRODUCTION}

Latent autoimmune diabetes in adults (LADA) is a subtype of autoimmune diabetes that accounts for $6-10 \%$ of all diabetes cases [1]. Clinical presentation is similar to that of type 2 diabetes (T2DM), while progression of $\beta$-cell destruction is slower than in type 1 diabetes (T1DM) [2]. The clinical profile $[1,3]$ and genetic profile [4-6] of LADA exhibits substantial overlap with those of both T1DM and T2DM, respectively, and LADA is therefore referred to as double diabetes or hybrid diabetes [7]. Universally acknowledged diagnostic criteria of LADA are age $\geq 30$ years with normal, high, or low body mass index (BMI), varying titers of autoimmune antibodies, and distinct degrees of $\beta$-cell destruction, insulin resistance, and metabolic syndrome $[8,9]$. These autoantibodies include glutamic acid decaroxylase antibody (GADA), insulin autoantibody, tyrosine phosphatase-related molecule- 2 autoantibody, zinc transporter-8 autoantibody [10-13], and the newly identified tetraspanin 7 autoantibody $[14,15]$. GADA is undoubtedly the most critical autoantibody in autoimmune diabetes [16]. In addition, the clinical characteristics of LADA differ markedly according to GADA titer (above or below $180 \mathrm{U} / \mathrm{ml})[17,18]$. LADA patients with high-titer GADA tend to be younger and leaner, with lower insulin secretion and with less frequent and severe metabolic syndrome [1].

Cellular autoimmunity is a major contributor to the development and progression of autoimmune diabetes. Patients with autoimmune diabetes have altered frequencies of immune cells, including T cells [19], B cells [20], natural killer cells [21], and dendritic cells [22]. These are generally considered to mediate the actual destruction of insulin-secreting $\beta$-cells in pancreatic islets. Among these, T helper type 1 (Th1) cells are the most critical players in many organ-specific autoimmune diseases, while T helper type 2 (Th2) cells are considered to be protective in terms of autoimmunity. However, this simple paradigm of Th1/Th2 imbalance is not sufficient to explain the immunopathology of different autoimmune diseases, such as autoimmune diabetes. The $\mathrm{T}$ helper type 17 (Th17) subset, which secretes the pro-inflammatory interleukin 17 (IL-17) cytokine, is considered to be a third member of effector $\mathrm{T}$ cells [23] that contribute to cell destruction in autoimmune diseases. Regulatory T cells (Tregs), which suppress the proliferation and cytokine secretion by $\mathrm{CD}^{+}$and $\mathrm{CD} 4^{+} \mathrm{T}$ cells, are critical suppressors in autoimmunity. High levels of IL-2 receptor $\alpha$-chain (CD25) and the transcription factor forkhead box protein 3 (FOXP3) are typically expressed in Tregs and so are used as specific markers for the differentiation of these cells. Expression of FOXP3 is reduced in T1DM [24] and LADA [25, 26]. Abnormal DNA methylation in $\mathrm{CD}^{+} \mathrm{T}$ cells is also involved in LADA progression [27].

Collectively, these findings suggest the presence of heterogeneous T-cell profiles and related transcription factors among patients with T1DM, DM, and LADA. However, few studies to date have compared T-cell subset profiles and the levels of associated transcription factors among these patients or between LADA patients with a high or low GADA titer. We speculated that high- and low-titer GADA-positive LADA patients would exhibit distinct T-cell phenotypes and differential expression of key transcription factors.

\section{METHODS}

\section{Patient Selection}

Patients with recent-onset LADA were recruited to the study based on the following criteria [9]: (1) diabetes diagnosed according to the 1999 
criteria of the World Health Organization (WHO; http://www.who.int/iris/handle/10665/ 66040), (2) age range from 30 to 70 years, (3) GADA positivity, (4) insulin independence for the first 6 months after diabetes diagnosis, and (5) diabetes duration of $\leq 3$ years. The exclusion criteria were: (1) evidence of any other autoimmune diseases, (2) evidence of chronic or acute infection, (3) history of any malignancy, congestive heart failure, or secondary diabetes; (4) in addition, women who were pregnant, who had frequent abortions, or who were breastfeeding were excluded.

Forty patients with LADA (median disease duration 21.0 [range 8.0-35.0] months) meeting the enrolment criteria were recruited from December 2014 to December 2016. Fourteen T2DM patients (median duration 7.0 [range 4.0-30.0] months) with negative GADA status were also enrolled in the study. Diagnostic criteria for T2DM were (1) hyperglycemia history according to WHO criteria, (2) negative for GADA, and (3) no requirement for immediate insulin treatment.

LADA and T2DM patients were selected from patients attending the outpatient clinic. Patients included in this study had similar lifestyle modifications. After a 3-month washing-in period, patients with a glycated hemoglobin (HbA1c) level of $<7.5 \%(58 \mathrm{mmol} / \mathrm{mol})$ were enrolled in a 1-month single insulin therapy run-in period. Sulfonylureas, insulin sensitizers, dipeptidyl peptidase 4 inhibition, and sodium-glucose cotransporter inhibitors were not administered to the patients in the study.

All procedures performed in studies involving human participants were in accordance with the 1964 Helsinki declaration and its later amendments or comparable ethical standards. The protocols were approved by the Ethics Committee of the Second Xiangya Hospital, Central South University. All patients were given written information on study procedures and goals, and provided informed consent. This study has been registered online (http://www. clinicaltrials. gov/identifier [NCT01159847]).

Physicians recorded height and weight. Fasting blood samples were tested for blood glucose (FBG), HbA1c, and blood C-peptide (FCP) levels. Two-hour postprandial blood samples were tested for blood glucose (PBG) and C-peptide (PCP) levels.

\section{GADA Assay}

Levels of GADA were measured by radioligand assay. this assay has been validated by the Diabetes Antibody Standardization Program (International Association for the Study of Pain [IASP] 2012) and was sponsored by the Immunology of Diabetes Society (IDS). The cutoff for GADA positivity was $18 \mathrm{U} / \mathrm{mL}$ (WHO units), and the sensitivity and specificity were 78 and $96.7 \%$, respectively. High-titer GADA was defined as a GADA titer of $\geq 180 \mathrm{U} / \mathrm{mL}$ [1].

\section{Th1/Th2/Th17/Treg Phenotype}

Fresh blood samples were collected into sodium heparin tubes from fasting subjects and processed within $2 \mathrm{~h}$. Peripheral blood mononuclear cells (PBMCs) were separated by Ficoll-Paque plus density-gradient centrifugation. Cells were stimulated with $50 \mathrm{ng} / \mathrm{mL}$ phorbol myristate acetate and $1 \mathrm{ug} / \mathrm{mL}$ ionomycin for $6 \mathrm{~h}$ in the presence of Brefeldin A and GolgiStop, following which the cells were harvested and stained with viability dye (Invitrogen, Carlsbad, CA, USA) and flurophoreconjugated anti-interferon gamma (anti-IFN- $\gamma$ ), anti-IL4, anti-IL17A, and anti-Foxp3. Cell numbers were counted using a Canto II flow cytometer (BD Biosciences, San Jose, CA, USA) and analyzed using FlowJo 7.6 software (Tree Star, Inc., Ashland, OR, USA). The gating strategy is shown in Fig. 1a, b.

\section{Quantification of mRNA Expression by Real-Time PCR}

Total RNA was obtained using TRIzol reagent (Invitrogen) and frozen at $-70^{\circ} \mathrm{C}$ until use. mRNA was first isolated with a mRNA extraction kit (Zymo Research, Irvine, CA, USA) and then reverse transcribed to complementary DNA (cDNA) using a cDNA Reverse Transcription Kit (GoScript; Promega, Madison, WI, USA) according to the manufacturer's instructions. The cDNAs were amplified using SYBR Green I 


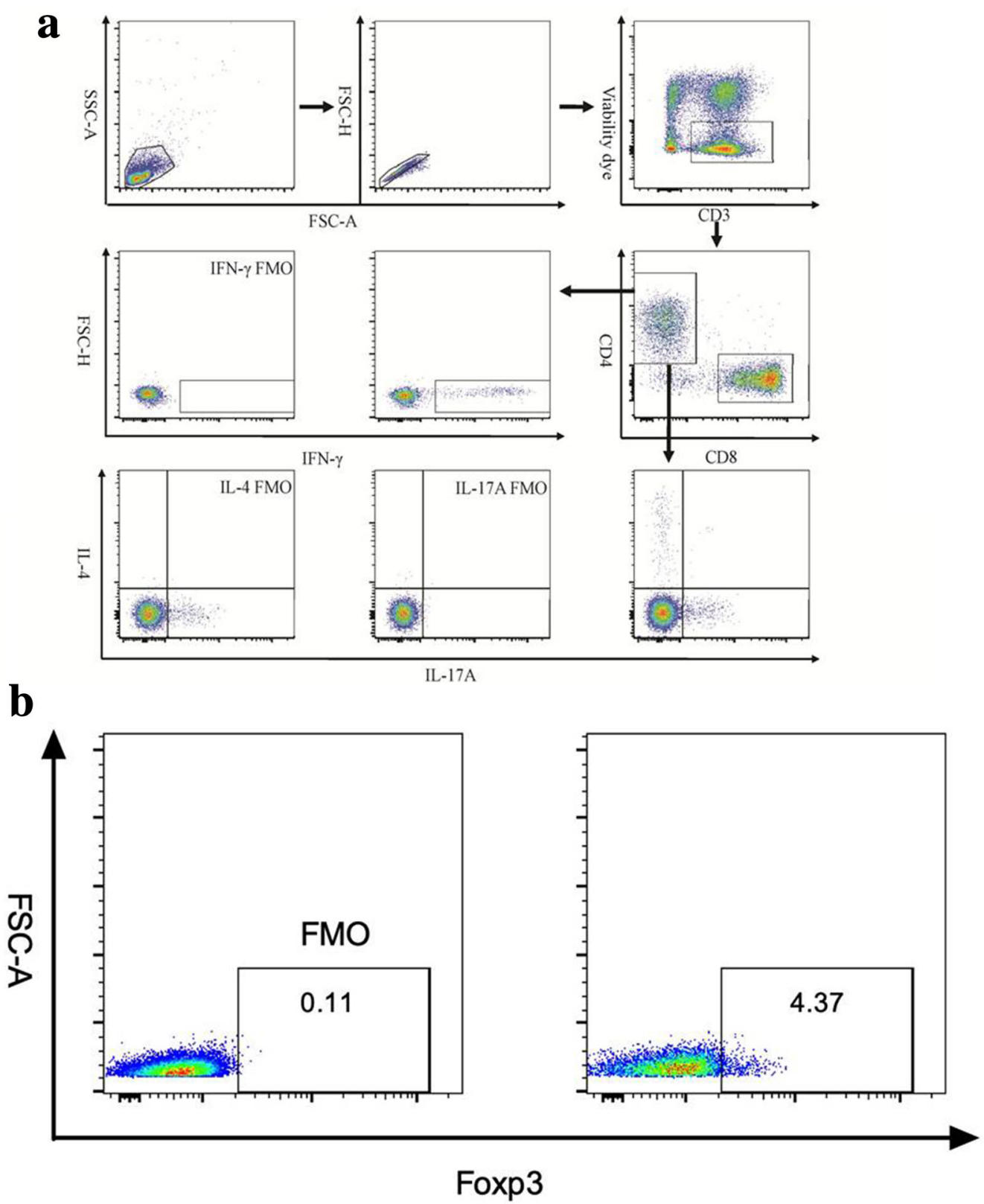

Fig. 1 a Representative plots and gating information of $\mathrm{T}$ helper type 1, 2 and 17 (Th1, Th2, and Th17, respectively) cells from the $\mathrm{T}$-cell fluorescence-activated cell sorting (FACS) analysis. Lymphocytes were first gated from peripheral blood mononuclear cells. The samples were analyzed using a BD FACS Canto II system and FlowJo

and specific oligonucleotides for the T-cell transcription factors T-bet (involved in Th1 differentiation), GATA3 (enriched in Th2 cells), FOXP3 (a major factor in Treg3), and RORC (Th17-enriched), and quantified by real-time cell analysis software (see text). FSC Forward scatter, FMO fluorescence Minus One control, INF interferon, IL interleukin, $S S C$ side scatter. b Representative plots and gating information on regulatory $\mathrm{T}$ ( Treg) cells from the T-cell FACS analysis

polymerase chain reaction (PCR) using an ABI PRISM Step One Sequence Detection System (PE Applied Biosystems, Foster City, CA, USA). The primers used in this study are listed in Table 1. The relative mRNA gene expression levels of all 
four T-cell transcription factors were quantified as fold-change relative to $\beta$-actin (internal control) using the $2^{-\Delta \Delta C T}$ method [28].

\section{Statistical Analysis}

Data are presented as mean \pm standard error of the mean or as the median with the 25 th-75th percentile, depending on the distribution. Means from normally distributed datasets were compared using Student's $t$ test. Non-parametric datasets were compared using the Mann-Whitney $U$ test. Multiple groups were compared by analysis of variance. Statistical analyses were conducted using SPSS software version 17.0 (IBM Corp., Armonk, NY, USA) and Prism5 software (GraphPad Software Inc., San Diego, CA, USA). Differences were considered to be significant at a two-tailed $P<0.05$.

\section{RESULTS}

\section{Group Differences in Demographic, Anthropometric, and Metabolic Parameters}

The clinical characteristics of the subjects in each group are summarized in Table 2. There were no significant differences in sex ratio, mean age, and BMI among LADA patients with low-titer GADA, LADA patients with high-titer GADA, and T2DM groups. However, disease duration was markedly shorter in the T2DM group. Compared to T2DM patients, LADA patients with higher-titer GADA (> $180 \mathrm{U} / \mathrm{mL})$ exhibited significantly higher PBG $(P=0.032)$, significantly lower FCP $(P=0.008)$, and significantly lower PCP $(P=0.006)$ levels. In LADA patients with low-titer GADA, the FCP level was reduced compared to patients with T2DM $(P=0.005)$.

\section{Differences in T-Cell Subset Frequencies Among LADA and T2DM Patients}

We initially analyzed the frequency (\%) of T-cell subsets in PBMCs by flow cytometry. No significant difference were found in the frequencies of Th1, Th2, Th17, and Treg cells between the LADA and T2DM groups (Fig. 2).

Surprisingly, LADA patients with high-titer GADA exhibited a greater Th1 cell frequency than did LADA patients low-titer GADA $(11.06 \pm 1.62$ vs. $7.05 \pm 0.86, P=0.030)$. The frequency of Th1 cells was also significantly reduced in the low-titer GADA group compared to the T2DM group $(7.05 \pm 0.86$ vs. $16.75 \pm 3.73, P=0.017$ ), while Th17 frequency was significant higher in the low-titer GADA group compared to the T2DM group $(1.11 \pm 0.09$ vs. $0.74 \pm 0.16, P=0.017)$ (Fig. 3).

\section{Differences in Transcription Factor mRNA Expression Levels Among LADA and T2DM Patients}

We next investigated expression levels of the $\mathrm{T}$ cell-specific transcription factors T-bet, GATA3, FOXP3, and RORC at the mRNA level in LADA and T2DM patients. Compared to the T2DM

Table 1 Forward and reverse primers for T-cell transcription factors

\begin{tabular}{lll}
\hline Target & Forward primer & Reverse primer \\
\hline T-bet & $5^{\prime}$-CAACGCTTCCAACACGCAT-3' & $5^{\prime}$-GACTCAAAGTTCTCCCGGAA-3' \\
GATA3 & $5^{\prime}$-TCATTAAGCCCAAGCGAAGG-3' & $5^{\prime}$-GTCCCCATTGGCATTCCTC-3' \\
RORC & $5^{\prime}$-GCAGCGCTCCAACATCTTCT-3' & $5^{\prime}$-ACGTACTGAATGGCCTCGGT-3' \\
FOXP3 & $5^{\prime}$-CACCTGGCTGGGAAAATGG-3' & $5^{\prime}$-GGAGCCCTTGTCGGATGA-3' \\
$\beta$-actin & $5^{\prime}$-CGGGAAATCGTGCGTGAC-3' & $5^{\prime}$-GGAAGGAAGGCTGGAAGAG-3' \\
\hline
\end{tabular}

See section "Quantification of mRNA Expression by Real-Time PCR" for explanation of targets 
Table 2 Metabolic characteristics of participants

\begin{tabular}{|c|c|c|c|}
\hline $\begin{array}{l}\text { Patient } \\
\text { characteristics }\end{array}$ & $\begin{array}{l}\text { LADA patients with high-titer } \\
\text { GADA }(n=19)\end{array}$ & $\begin{array}{l}\text { LADA patients with low-titer } \\
\text { GADA }(n=21)\end{array}$ & T2DM $(n=14)$ \\
\hline Sex (female/male) & $9 / 10$ & $10 / 11$ & $6 / 8$ \\
\hline Age (years) & $50.8 \pm 12.4$ & $48.1 \pm 11.7$ & $53.6 \pm 9.5$ \\
\hline $\begin{array}{l}\text { Duration of disease } \\
\text { (months) }\end{array}$ & $21.0[7.0-29.0]$ & $20.0[8.0-36.0]$ & $7.0[4.0-30.0]$ \\
\hline $\operatorname{BMI}\left(\mathrm{kg} / \mathrm{m}^{2}\right)$ & $24.1 \pm 3.6$ & $24.0 \pm 2.8$ & $22.4 \pm 2.7$ \\
\hline $\mathrm{FBG}(\mathrm{mmol} / \mathrm{L})$ & $7.06 \pm 2.03$ & $7.10 \pm 2.49$ & $6.08 \pm 1.19$ \\
\hline $\mathrm{PBG}(\mathrm{mmol} / \mathrm{L})$ & $13.84 \pm 5.59^{*}$ & $12.38 \pm 4.92$ & $10.39 \pm 3.13$ \\
\hline GADA $(\mathrm{U} / \mathrm{ml})$ & $623.4[532.0-853.6]^{\Delta \Delta}$ & $70.4[27.6-86.7]$ & - \\
\hline $\begin{array}{l}\mathrm{HbAlc} \% \\
\qquad(\mathrm{mmol} / \mathrm{mol})\end{array}$ & $6.6 \pm 0.9(47.09 \pm 3.03)$ & $6.6 \pm 1.7(48.07 \pm 1.92)$ & $\begin{array}{l}6.0 \pm 0.5 \\
\quad(45.53 \pm 1.02)\end{array}$ \\
\hline FCP $(\mathrm{pmol} / \mathrm{L})$ & $352.7[300.4-629.3]^{* *}$ & $451.8[299.6-594.6]^{* *}$ & $\begin{array}{l}593.3 \\
{[542.2-798.3]}\end{array}$ \\
\hline PCP $(\mathrm{pmol} / \mathrm{L})$ & $1359.9[933.4-1798.1]^{* *}$ & $1578.9[866.1-2174.0]$ & $\begin{array}{l}1858.1 \\
\quad[1482.0-2909.6]\end{array}$ \\
\hline
\end{tabular}

${ }^{*} P<0.05$, compared with T2DM group. ${ }^{* *} P<0.01$, compared to T2DM. ${ }^{\Delta \Delta} P<0.01$, compared to low-titer GADA LADA patients

Data are presented as the median with the 25 th -75 th percentile in square brackets or as the mean \pm standard error of the mean (SEM)

$B M I$ Body mass index, $F B G$ fasting blood glucose, $F C P$ fasting C-peptide, $G A D A$ glutamic acid decarboxylase autoantibody, HbA1c glycated hemoglobin, $L A D A$ latent autoimmune diabetes in adults, PCP 2-h postprandial C-peptide, PBG 2-h postrandial blood glucose, $T 2 D M$ type 2 diabetes mellitus

group $(n=14)$, the whole LADA group $(n=40)$ demonstrated lower mRNA expression levels of all four transcription factors, but the differences did not reach significance (Fig. 4).

There were, however, multiple significant differences when groups were compared following the separation of LADA patients into low- and high-titer GADA groups (Fig. 5). Compared to the low-titer GADA group, the high-titer group expressed significantly reduced levels of FOXP3 mRNA and RORC mRNA $(0.35 \pm 0.13 \quad$ vs. $\quad 1.50 \pm 0.41, \quad P=0.027$ $0.53 \pm 0.19$ vs. $1.35 \pm 0.21, P=0.027$, respectively), while no significant differences were found in T-bet and GATA3 mRNA expression between the two LADA groups. Compared to the T2DM group, LADA patients in the hightiter GADA group also presented reduced expression levels of FOXP3 mRNA $(0.35 \pm 0.13$ vs. $1.75 \pm 0.54, P=0.002)$, RORC $(0.53 \pm 0.19$ vs. $2.00 \pm 0.77, \quad P=0.046), \quad$ and GATA3 $(0.74 \pm 0.17$ vs. $2.31 \pm 0.91, P=0.046)$, while no significant differences in T-bet, GATA3, FOXP3 and RORC mRNA expression levels were found between the low-titer GADA group and the T2DM group (Fig. 5).

\section{Correlation of T-Cell Subsets and Relevant Transcription Factors with Metabolic Parameters}

We then examined whether these alterations in T-cell subset profiles were associated with clinical parameters. The proportions of T-cell subsets did not correlate significantly with age, 


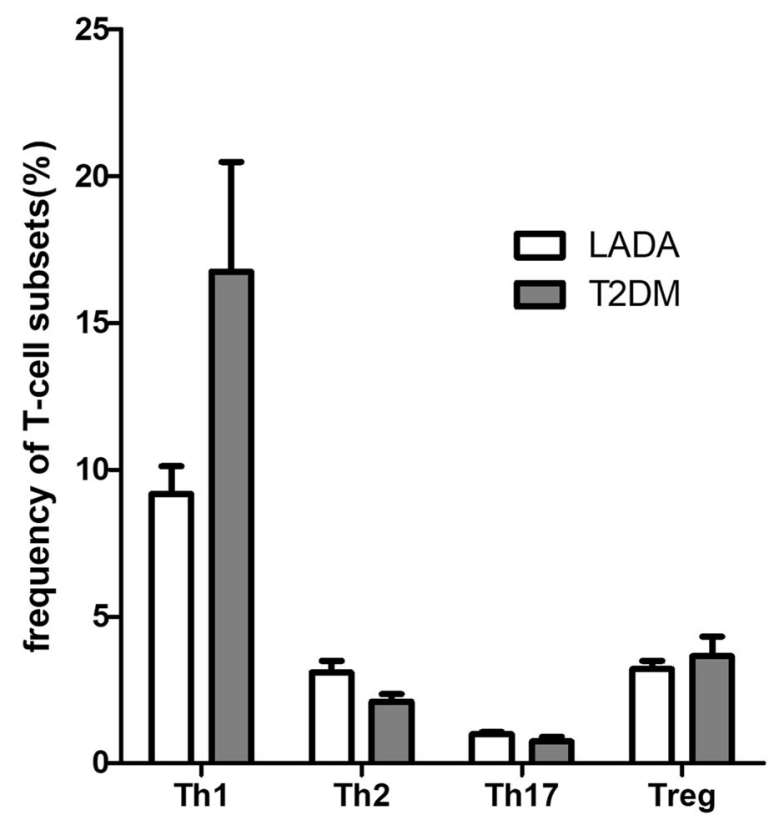

Fig. 2 Frequencies of $\mathrm{T}$-cell subsets (Th1, Th2, Th17, Treg) in the type 2 diabetes mellitus $(T 2 D M)$ and latent autoimmune diabetes in adults $(L A D A)$ groups of patients. $T h 1, T h 2, T h 17$ T-helper cell types $1,2,17$, respectively

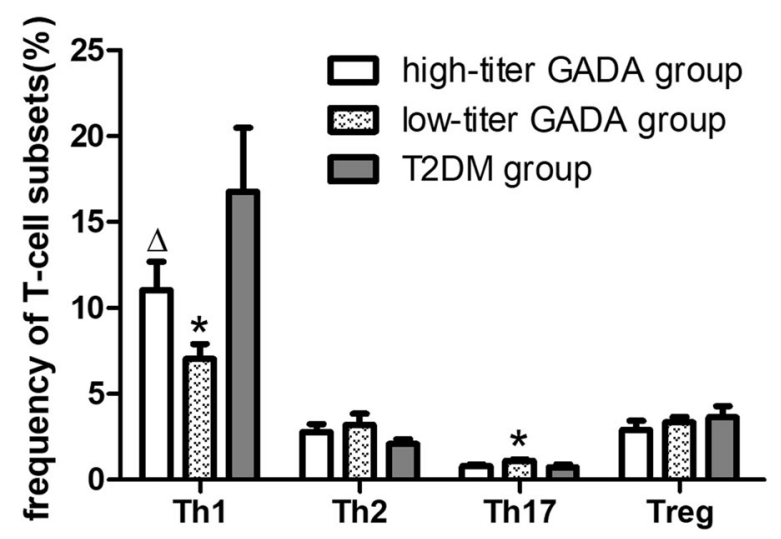

Fig. 3 Frequencies of T-cell subsets among LADA patients with high-titer glutamic acid decarboxylase autoantibody $(G A D A)$, LADA patients with low-titer GADA, and T2DM groups. ${ }^{*} P<0.05$ compared to T2DM, ${ }^{\Delta} P<0.05$ compared to LADA patients with low-titer GADA

disease duration, FBG, FCP, HbA1c, or GADA titer. Further, there were no significant correlations between transcription factor expression levels and age, duration, FBG, FCP, HbA1c, or GADA titer. However, there was a weak negative

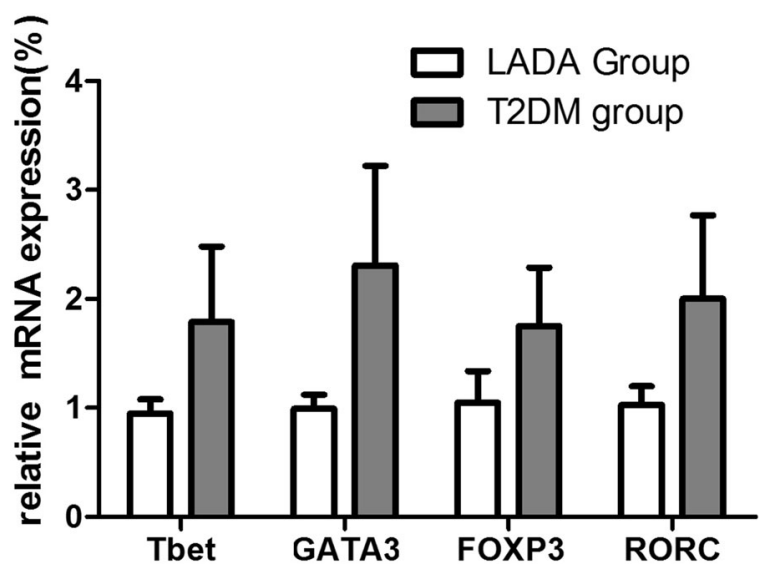

Fig. 4 Relative mRNA expression levels of the $T$ cellspecific transcription factors T-bet, GATA3, FOXP3, and RORC in LADA patients $(n=40)$, and T2DM patients $(n=14)$ as measured by real-time PCR. Levels of target gene mRNA transcripts are normalized to $\beta$-actin

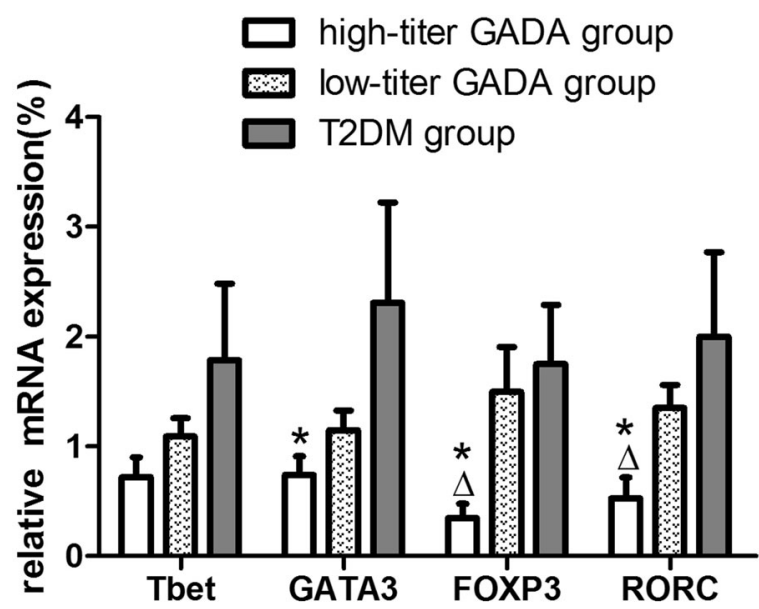

Fig. 5 Relative mRNA expression levels of the $\mathrm{T}$ cellspecific transcription factors T-bet, GATA3, FOXP3, and RORC in LADA patients with high-titer GADA $(n=19)$, LADA patients with low-titer GADA $(n=21)$, and T2DM patients $(n=14)$ as measured by real-time PCR. Levels of target gene mRNA transcripts are normalized to $\beta$-actin. ${ }^{*} P<0.05$ compared to T2DM, ${ }^{\Delta} P<0.05$ compared to low-titer GADA patients with LADA

correlation between FOXP3 expression and GADA titer for the entire cohort $(r=-0.0433$, $P=0.015$ ), suggesting some association within a specific group. Indeed there were strong negative correlations between GADA titer and both 
FOXP3 $(r=-0.606, P=0.008)$ and RORC $(r=$ $-0.536, P=0.022)$ in the LADA group. Alternatively, there were no significant correlations between T-cell subsets and age, duration, FBG, FCP, PBG, PCP, HbA1c, or GADA titer.

\section{DISCUSSION}

Latent autoimmune diabetes in adults is an autoimmune disorder associated with multiple environmental, lifestyle, and genetic factors. Previous studies $[1,17,29,30]$ have confirmed that LADA patients with high-titer GADA tend to be younger and leaner and have lower insulin secretion, poorer blood glycemic control, and less frequent metabolic syndrome than do LADA patients with low-titer GADA [1], while low-titer GADA patients have demographic and disease characteristics more similar to T2DM. Furthermore, high titers of antigen-specific islet antibodies can predict the risk of future $\beta$-cell failure and increasing insulin requirements $[18,31]$. Thus, LADA patients possess a broad and heterogeneous clinical phenotype that depends in part on GADA titer. In our study, LADA patients showed poorer blood glucose control and lower PCP level than did T2DM patients, in accordance with results from previous studies [1, 29].

There is compelling evidence that $\mathrm{T}$ cells are major contributors to the immunopathogenesis of autoimmune diabetes. Nevertheless, the underlying mechanisms are still not completely clarified, especially in LADA patients with high or low GADA titer. The main goal of this study was to investigate the differences in T-cell subset frequencies and expression levels of subsetspecific or -enriched transcription factors in LADA patients with high or low GADA titer.

The canonical Th1/Th2 paradigm is not sufficient to explain the immunopathology of autoimmune diseases, such as autoimmune diabetes. Th17 cells, which mainly secrete the pro-inflammatory cytokine IL-17, participate in $\beta$-cell destruction. Th17 and Th1 cells are considered to be critical elements at different phases of the autoimmune process [32]. Treg cells, the most important regulators, suppress T-cell proliferation and maintain immune system balance. In our study, we found no significant differences in the frequencies of Th1, Th2, Treg, and Th17 between the LADA groups. Surprisingly, the frequency of Th1 cells was lower and that of Th17 cells higher in the low-titer GADA group than in the T2DM group. However, this difference was also found between the high-titer GADA group and theT2DM group. In addition, Th1 frequency was significantly elevated in the high-titer GADA group compared to the lowtiter GADA group. These differences were not paralleled by expression of the corresponding subset-specific T-cell transcription factors, suggesting no functional differences in T cells.

Previous studies [33-35] have reported a reduced Th1-mediated immune response, while a more recent study [24] confirmed lower expression of $\mathrm{T}$ cell-specific transcription factors during the autoimmune process. In our study as well, T cell-specific transcription factor expression was lower in LADA patients than T2DM patients, although the difference did not reach significance. When LADA patients were separated into high-titer and low-titer GADA groups, differences in transcription factor expression emerged, in line with previous studies and suggesting a possible exhaustion effect due to chronic activation of the immune system, especially among high-titer GADA patients. The exact phenotype and function of these putative exhausted cells, especially in high-titer GADA patients with LADA, will be investigated in a future study. FOXP3 is considered to be crucial to Treg development and function [36], and several previous studies have reported significantly lower expression in LADA patients compared to T2DM patients $[23,24]$. In the current study as well, we found that FOXP3 expression was lower in LADA patients than in T2DM patients, although the difference did not reach significance. Surprisingly, there were significant declines in the mRNA expression levels of GATA3, FOXP3, and RORC in the high-titer GADA LADA group compared to the T2DM group, which is consistent with results reported from previous studies, as well a non-significant decrease in T-bet expression. Furthermore, we also found decreased expression of FOXP3 and RORC in the high-titer LADA group compared to the low-titer LADA group. There was also a negative 
correlation between the expression of FOXP3 and GADA titer. These results suggest that GADA titer may reflect the severity of cellular immune destruction, with higher titer in LADA patients indicating greater immune-mediated damage. LADA patients with high-titer GADA tend to have more serious immune state and a poorer $\beta$-cell function and glycemic control.

In conclusion, our study reveals several intrinsic differences in the immunologic profile of T-cell subsets between LADA and T2DM and further differences between LADA patients with a high or low GADA titer. We also provide the first report of significantly lower expression levels of T-cell transcription factors in LADA patients versus those with T2DM as well as lower expression of FOXP3 in LADA patients with high-titer GADA versus LADA patients with low-titer GADA. Further, GADA titer was negatively correlated with FOXP3 expression, indicating more severe immune destruction among LADA patients with high-titer GADA. These differences may be crucial for understanding the immunopathogenesis of diabetes in LADA patients. These findings also provide potential molecular targets for more effective clinical treatment of LADA with high-titer GADA.

Further investigation in a larger cohort is warranted to assess clinical outcomes as well as to more thoroughly explore the possible immunoregulation mechanism of T-cells and associated transcription factors in these patients.

\section{ACKNOWLEDGEMENTS}

We thank all the participants of the study.

Funding. This work was sponsored by the National Key R\&D Program of China (2018YFC 1315603). No funding or sponsorship was received for the publication of this article. The article processing charges were funded by the authors.

Editorial Assistance. Guangzhou Liheng Translation Consulting Co. LTD provided editorial support and English language support prior to submission.

Authorship. All named authors meet the International Committee of Medical Journal Editors (ICMJE) criteria for authorship for this article, take responsibility for the integrity of the work as a whole, and have given their approval for this version to be published.

Disclosures. Xia Wang, Lin Yang, Ying Cheng, Huiying Liang, Jingping $\mathrm{Hu}$, Peilin Zheng, Gan Huang and Zhiguang Zhou declare no conflicts of interest.

Compliance with Ethics Guidelines. All procedures performed in studies involving human participants were in accordance with the 1964 Helsinki declaration and its later amendments or comparable ethical standards. The protocols were approved by the Ethics Committee of the Second Xiangya Hospital, Central South University. All patients were given written information on study procedures and goals, and provided informed consent. This study has been registered online (http://www. clinicaltrials. gov/identifier [NCT01159847]).

Data Availability. The datasets during and/ or analyzed during the current study are available from the corresponding author on reasonable request.

Open Access. This article is distributed under the terms of the Creative Commons Attribution-NonCommercial 4.0 International License (http://creativecommons.org/licenses/ by-nc/4.0/), which permits any noncommercial use, distribution, and reproduction in any medium, provided you give appropriate credit to the original author(s) and the source, provide a link to the Creative Commons license, and indicate if changes were made.

\section{REFERENCES}

1. Zhou Z, Xiang Y, Ji L, et al. Frequency, immunogenetics, and clinical characteristics of latent autoimmune diabetes in China (LADA China 
study): a nationwide, multicenter, clinic-based cross-sectional study. Diabetes. 2013;62(2):543-50.

2. Naik RG, Brooks-Worrell BM, Palmer JP. Latent autoimmune diabetes in adults. J Clin Endocrinol Metab. 2009;94(12):4635-44.

3. Leslie RD, Kolb H, Schloot NC, et al. Diabetes classification: grey zones, sound and smoke: action LADA 1. Diabetes Metab Res Rev. 2008;24(7):511-9.

4. Nambam B, Aggarwal S, Jain A. Latent autoimmune diabetes in adults: a distinct but heterogeneous clinical entity. World J Diabetes. 2010;1(4):111-5.

5. Cervin C, Lyssenko V, Bakhtadze E, et al. Genetic similarities between latent autoimmune diabetes in adults, type 1 diabetes, and type 2 diabetes. Diabetes. $2008 ; 57(5): 1433-7$.

6. Zheng P, Kissler S. PTPN22 silencing in the NOD model indicates the type 1 diabetes-associated allele is not a loss-of-function variant. Diabetes. 2013;62(3):896-904.

7. Pozzilli P, Guglielmi C, Pronina E, et al. Double or hybrid diabetes associated with an increase in type 1 and type 2 diabetes in children and youths. Pediatr Diabetes. 2007;8[Suppl 9]:88-95.

8. Cernea S, Buzzetti R, Pozzilli P. Beta-cell protection and therapy for latent autoimmune diabetes in adults. Diabetes Care. 2009;32[Suppl 2]:S246-52.

9. Fourlanos S, Dotta F, Greenbaum CJ, et al. Latent autoimmune diabetes in adults (LADA) should be less latent. Diabetologia. 2005;48(11):2206-12.

10. Yi B, Huang G, Zhou Z. Different role of zinc transporter 8 between type 1 diabetes mellitus and type 2 diabetes mellitus. J Diabetes Investig. 2016;7(4):459-65.

11. Huang G, Xiang Y, Pan L, et al. Zinc transporter 8 autoantibody (ZnT8A) could help differentiate latent autoimmune diabetes in adults (LADA) from phenotypic type 2 diabetes mellitus. Diabetes Metab Res Rev. 2013;29(5):363-8.

12. Arvan P, Pietropaolo M, Ostrov D, et al. Islet autoantigens: structure, function, localization, and regulation. Cold Spring Harb Perspect Med. 2012;2(8):a007658.

13. Gohlke H, Ferrari U, Koczwara K, et al. SLC30A8 (ZnT8) polymorphism is associated with young age at type 1 diabetes onset. Rev Diabet Stud. 2008;5(1):25-7.

14. Walther D, Eugster A, Jergens S, et al. Tetraspanin 7 autoantibodies in type 1 diabetes. Diabetologia. 2016;59(9):1973-6.
15. McLaughlin KA, Richardson CC, Ravishankar A, et al. Identification of tetraspanin-7 as a target of autoantibodies in type 1 diabetes. Diabetes. 2016;65(6):1690-8.

16. Xiang Y, Huang G, Shan Z, et al. Glutamic acid decarboxylase autoantibodies are dominant but insufficient to identify most Chinese with adultonset non-insulin requiring autoimmune diabetes: LADA China study 5. Acta Diabetol. 2015;52(6):1121-7.

17. Buzzetti R, Di Pietro S, Giaccari A, et al. High titer of autoantibodies to GAD identifies a specific phenotype of adult-onset autoimmune diabetes. Diabetes Care. 2007;30(4):932-8.

18. Zampetti S, Campagna G, Tiberti C, et al. High GADA titer increases the risk of insulin requirement in LADA patients: a 7-year follow-up (NIRAD study 7). Eur J Endocrinol. 2014;171(6):697-704.

19. Kaaba SA, Al-Harbi SA. Abnormal lymphocyte subsets in Kuwaiti patients with type-1 insulin-dependent diabetes mellitus and their first-degree relatives. Immunol Lett. 1995;47(3):209-13.

20. Deng C, Xiang Y, Tan T, et al. Altered peripheral B-lymphocyte subsets in type 1 diabetes and latent autoimmune diabetes in adults. Diabetes Care. 2016;39(3):434-40.

21. Akesson C, Uvebrant K, Oderup C, et al. Altered natural killer (NK) cell frequency and phenotype in latent autoimmune diabetes in adults (LADA) prior to insulin deficiency. Clin Exp Immunol. 2010;161(1):48-56.

22. Nieminen JK, Vakkila J, Salo HM, et al. Altered phenotype of peripheral blood dendritic cells in pediatric type 1 diabetes. Diabetes Care. 2012;35(11):2303-10.

23. Bettelli E, Korn T, Kuchroo VK. Th17: the third member of the effector $\mathrm{T}$ cell trilogy. Curr Opin Immunol. 2007;19(6):652-7.

24. Hamari S, Kirveskoski T, Glumoff V, et al. Analyses of regulatory CD4 + CD25 + FOXP3 + T cells and observations from peripheral $\mathrm{T}$ cell subpopulation markers during the development of type 1 diabetes in children. Scand J Immunol. 2016;83(4):279-87.

25. Yang Z, Zhou Z, Huang G, et al. The CD4(+) regulatory $\mathrm{T}$-cells is decreased in adults with latent autoimmune diabetes. Diabetes Res Clin Pract. 2007;76(1):126-31.

26. Radenkovic M, Silver C, Arvastsson J, et al. Altered regulatory $\mathrm{T}$ cell phenotype in latent autoimmune diabetes of the adults (LADA). Clin Exp Immunol. 2016;186(1):46-56. 
27. Li Y, Zhao M, Hou C, et al. Abnormal DNA methylation in $\mathrm{CD} 4+\mathrm{T}$ cells from people with latent autoimmune diabetes in adults. Diabetes Res Clin Pract. 2011;94(2):242-8.

28. Livak KJ, Schmittgen TD. Analysis of relative gene expression data using real-time quantitative PCR and the 2(-Delta Delta C(T)) Method. Methods. 2001;25(4):402-8.

29. Liu L, Li X, Xiang Y, et al. Latent autoimmune diabetes in adults with low-titer GAD antibodies: similar disease progression with type 2 diabetes: a nationwide, multicenter prospective study (LADA China Study 3). Diabetes Care. 2015;38(1):16-21.

30. van Deutekom AW, Heine RJ, Simsek S. The islet autoantibody titres: their clinical relevance in latent autoimmune diabetes in adults (LADA) and the classification of diabetes mellitus. Diabet Med. 2008;25(2):117-25.

31. Borg H, Gottsater A, Landin-Olsson M, et al. High levels of antigen-specific islet antibodies predict future beta-cell failure in patients with onset of diabetes in adult age. J Clin Endocrinol Metab. 2001;86(7):3032-8.
32. Li CR, Mueller EE, Bradley LM. Islet antigen-specific Th17 cells can induce TNF-alpha-dependent autoimmune diabetes. J Immunol. 2014;192(4):1425-32.

33. Tsiavou A, Degiannis D, Hatziagelaki E, et al. Intracellular IFN-gamma production and IL-12 serum levels in latent autoimmune diabetes of adults (LADA) and in type 2 diabetes. J Interferon Cytokine Res. 2004;24(7):381-7.

34. Ryden A, Ludvigsson J, Fredrikson M, et al. General immune dampening is associated with disturbed metabolism at diagnosis of type 1 diabetes. Pediatr Res. 2014;75(1-1):45-50.

35. Foss NT, Foss-Freitas MC, Ferreira MA, et al. Impaired cytokine production by peripheral blood mononuclear cells in type 1 diabetic patients. Diabetes Metab. 2007;33(6):439-43.

36. Sakaguchi S, Miyara M, Costantino CM, et al. FOXP3 + regulatory T cells in the human immune system. Nat Rev Immunol. 2010;10(7):490-500. 